\title{
817.
}

\section{ON THE SIXTEEN-NODAL QUARTIC SURFACE.}

[From Crelle's Journal der Mathem., t. XcIv. (1883), pp. 270-272.]

Riemann's theory of the bitangents of a plane quartic leads at once to a very simple form of the equation of the sixteen-nodal surface: viz. if $\xi, \eta, \zeta$ denote linear functions of the coordinates $(x, y, z, w)$ such that identically

$$
\begin{aligned}
x+y+z+\xi+\eta+\zeta & =0, \\
a x+b y+c z+f \xi+g \eta+h \zeta & =0,
\end{aligned}
$$

(where $a f=b g=c h=1$ ), then the quartic surface

$$
\sqrt{x \xi}+\sqrt{y \eta}+\sqrt{z \zeta}=0
$$

has the sixteen singular tangent planes (each touching it along a conic)

$$
\begin{aligned}
x=0, & y=0, \quad z=0, \quad \xi=0, \quad \eta=0, \quad \zeta=0, \\
x+y+z=0, & a x+b y+c z=0, \\
\xi+y+z=0, & f \xi+b y+c z=0, \\
x+\eta+z=0, & a x+g \eta+c z=0, \\
x+y+\zeta=0, & a x+b y+h \zeta=0, \\
\frac{x}{1-b c}+\frac{y}{1-c a}+\frac{z}{1-a b}=0, & \frac{\xi}{1-g h}+\frac{\eta}{1-h f}+\frac{\zeta}{1-f g}=0:
\end{aligned}
$$

and it is thus a sixteen-nodal surface.

I have formerly given the equation of this surface under the form

$$
\sqrt{x(X-w)}+\sqrt{y(Y-w)}+\sqrt{z(Z-w)}=0,
$$


where

$$
\begin{aligned}
& \alpha+\beta+\gamma=0, X=\alpha\left(\gamma^{\prime} \gamma^{\prime \prime} y-\beta^{\prime} \beta^{\prime \prime} z\right), \\
& \alpha^{\prime}+\beta^{\prime}+\gamma^{\prime}=0, Y=\beta\left(\alpha^{\prime} \alpha^{\prime \prime} z-\gamma^{\prime} \gamma^{\prime \prime} x\right), \\
& \alpha^{\prime \prime}+\beta^{\prime \prime}+\gamma^{\prime \prime}=0, Z=\gamma\left(\beta^{\prime} \beta^{\prime \prime} x-\alpha^{\prime} \alpha^{\prime \prime} y\right), \\
& P=\frac{x}{\alpha}+\frac{y}{\beta}+\frac{z}{\gamma}, X^{\prime}=\alpha^{\prime}\left(\gamma^{\prime \prime} \gamma y-\beta^{\prime \prime} \beta z\right), \\
& P^{\prime}=\frac{x}{\alpha^{\prime}}+\frac{y}{\beta^{\prime}}+\frac{z}{\gamma^{\prime}}, Y^{\prime}=\beta^{\prime}\left(\alpha^{\prime \prime} \alpha z-\gamma^{\prime \prime} \gamma x\right), \\
& P^{\prime \prime}=\frac{x}{\alpha^{\prime \prime}}+\frac{y}{\beta^{\prime \prime}}+\frac{z}{\gamma^{\prime \prime}}, Z^{\prime}=\gamma^{\prime}\left(\beta^{\prime \prime} \beta x-\alpha^{\prime \prime} \alpha y\right), \\
& X^{\prime \prime}=\alpha^{\prime \prime}\left(\gamma \gamma^{\prime} y-\beta \beta^{\prime} z\right), \\
& Y^{\prime \prime}=\beta^{\prime \prime}\left(\alpha \alpha^{\prime} z-\gamma \gamma^{\prime} x\right), \\
& Z^{\prime \prime}=\gamma^{\prime \prime}\left(\beta \beta^{\prime} x-\alpha \alpha^{\prime} y\right),
\end{aligned}
$$

and where the equations of the sixteen singular tangent planes are

$$
\begin{array}{rrrr}
x=0, & y=0, & z=0, & w=0 \\
X-w=0, & Y-w=0, & Z-w=0, & P=0 \\
X^{\prime}-w=0, & Y^{\prime}-w=0, & Z^{\prime}-w=0, & P^{\prime}=0 \\
X^{\prime \prime}-w=0, & Y^{\prime \prime}-w=0, & Z^{\prime \prime}-w=0, & P^{\prime \prime}=0 ;
\end{array}
$$

see Crelle's Journal, vol. LxxiII. (1871), pp. 292, 293, [442], and also Proc. Lond. Math. Soc., vol. III. (1871), p. 251*, [454].

To identify the two forms, using $x^{\prime}, y^{\prime}, z^{\prime}, \xi^{\prime}, \eta^{\prime}, \zeta^{\prime}$ for the new form, I assume

$$
x^{\prime}, y^{\prime}, z^{\prime}, \xi^{\prime}, \eta^{\prime}, \zeta^{\prime}=l x, m y, n z, p(X-w), q(Y-w), r(Z-w),
$$

where $l p=m q=n r=1$; and so convert the equation

into

$$
\sqrt{x(X-w)}+\sqrt{y(Y-w)}+\sqrt{z(Z-w)}=0
$$

$$
\sqrt{x^{\prime} \xi^{\prime}}+\sqrt{y^{\prime} \eta^{\prime}}+\sqrt{z^{\prime} \zeta^{\prime}}=0 .
$$

The constants $(l, m, n, p, q, r)$ and $(a, b, c, f, g, h)$, where $a f=b g=c h=1$, are then to be determined so that we may have identically

$$
\begin{aligned}
x^{\prime}+y^{\prime}+z^{\prime}+\xi^{\prime}+\eta^{\prime}+\zeta^{\prime} & =0 \\
a x^{\prime}+b y^{\prime}+c z^{\prime}+f \xi^{\prime}+g \eta^{\prime}+h \zeta^{\prime} & =0
\end{aligned}
$$

and we thus obtain 8 new equations to be satisfied by the 12 constants, viz. these are

$$
\begin{aligned}
& l+r \cdot \gamma \beta^{\prime} \beta^{\prime \prime}-q \cdot \beta \gamma^{\prime} \gamma^{\prime \prime}=0 \\
& m+p \cdot \alpha \gamma^{\prime} \gamma^{\prime \prime}-r \cdot \gamma \alpha^{\prime} \alpha^{\prime \prime}=0 \\
& n+q \cdot \beta \alpha^{\prime} \alpha^{\prime \prime}-p \cdot \alpha \beta^{\prime} \beta^{\prime \prime}=0 \\
& p+q+r \quad=0 \\
& a l+h r \cdot \gamma \beta^{\prime} \beta^{\prime \prime}-g q \cdot \beta \gamma^{\prime} \gamma^{\prime \prime}=0 \\
& b m+f p \cdot \alpha \gamma^{\prime} \gamma^{\prime \prime}-h r \cdot \gamma \alpha^{\prime \prime} \alpha^{\prime \prime}=0 \\
& c n+g q \cdot \beta \alpha^{\prime} \alpha^{\prime \prime}-f p \cdot \alpha \beta^{\prime} \beta^{\prime \prime}=0 \\
& f p+g q+h r \quad=0
\end{aligned}
$$

[* This Collection, vol, viI., p. 282.] 
But substituting for $a, b, c, l, m, n$ their values $\frac{1}{f}, \frac{1}{g}, \frac{1}{h}, \frac{1}{p}, \frac{1}{q}, \frac{1}{r}$, we have in all 8 equations for the determination of $q r, r p, p q, g h, h f, f g$; viz. if for greater convenience we introduce the new symbols $\mathfrak{A}, \mathfrak{B}, \quad \widetilde{C}=q r \alpha^{\prime} \alpha^{\prime \prime}, r p \beta^{\prime} \beta^{\prime \prime}, p q \gamma^{\prime} \gamma^{\prime \prime}$, then the 8 equations are

$$
\begin{aligned}
& \frac{1}{\beta \gamma}+\frac{\mathfrak{B}}{\beta}-\frac{\mathfrak{S}}{\gamma}=0 \\
& \frac{1}{\gamma \alpha}+\frac{\mathfrak{S}}{\gamma}-\frac{\mathfrak{A}}{\alpha}=0 \\
& \frac{1}{\alpha \beta}+\frac{\mathfrak{A}}{\alpha}-\frac{\mathfrak{B}}{\beta}=0 \\
& \frac{\alpha^{\prime} \alpha^{\prime \prime}}{\mathfrak{A}}+\frac{\beta^{\prime} \beta^{\prime \prime}}{\mathfrak{B}}+\frac{\gamma^{\prime} \gamma^{\prime \prime}}{\mathfrak{\delta}}=0 \\
& \frac{1}{\beta \gamma}+h f \cdot \frac{\mathfrak{B}}{\beta}-f g \cdot \frac{(5}{\gamma}=0 \\
& \frac{1}{\gamma \alpha}+f g \cdot \frac{\mathfrak{\zeta}}{\gamma}-g h \cdot \frac{\mathfrak{A}}{\alpha}=0 \\
& \frac{1}{\alpha \beta}+g h \cdot \frac{\mathfrak{A}}{\alpha}-h f \cdot \frac{\mathfrak{B}}{\beta}=0 \\
& \frac{\alpha^{\prime} \alpha^{\prime \prime}}{\mathfrak{A} g h}+\frac{\beta^{\prime} \beta^{\prime \prime}}{\mathfrak{B} h f}+\frac{\gamma^{\prime} \gamma^{\prime \prime}}{\mathfrak{S} f g}=0
\end{aligned}
$$

But in virtue of the equation $\alpha+\beta+\gamma=0$ the first four equations are equivalent to three equations only, and they determine $\mathfrak{A}, \mathfrak{B}$, $\mathfrak{\complement}$, that is, $p, q, r$, which give at once $l, m, n$; and similarly the second four equations are equivalent to three equations only, and $\mathfrak{I}, \mathfrak{B}$, $\mathfrak{C}$ being known they determine $g h, h f, f g$, that is, $f, g$, $h$, which give at once $a, b, c$ : the identification of the two forms is thus completed.

Cambridge, 11th January, 1883.

C. XII. 